\title{
An Image Rotation Approach for Hand Dorsal Vein Recognition
}

\author{
Ignacio Irving Morales-Montiel, J. Arturo Olvera-López, Ivan Olmos-Pineda \\ Benemerita Universidad Autonoma de Puebla, \\ Facultad de Ciencias de la Computacion, Puebla, Pue., \\ Mexico \\ cpycon@hotmail.com, $\{$ aolvera,iolmos $\} @$ cs.buap.mx
}

\begin{abstract}
In Biometric recognition, commonly the information about the biometric to be analyzed is contained in digital images, in particular this work is focused on analyzing hand dorsal veins as biometric. A basic process during the recognition stage is the feature (minutiae) extraction; when images are captured from several people it is very difficult to obtain a global alignment and as consequence different orientation images are obtained which makes more difficult the feature extraction. In this work we propose an approach based on central hand points for auto-rotating hand thermal images; based on the results our approach is able to rotate images from different orientations and obtains homogeneous alignment of the tested images.
\end{abstract}

Keywords: Hand dorsal thermal image rotation, segmentation, biometric systems, hand vein recognition.

\section{Introduction}

Nowadays several aspects in the real life are aided by computer technology; in particular some difficult tasks for humans can be solved using computer systems. Mainly, the difficult tasks for humans are related to those problems involving huge amounts of information to be analyzed in short periods of time. As a particular case of this kind of difficult human tasks, we can state the person recognition problem in public places (during the whole day i.e. twenty four hours) where the goal is the identification of at least one person in a scenario in which there exists not only the person to identify but also other people and some other objects.

In Computer Science, there exists an area which is focused on solving the person recognition problem, this area is named Biometric Recognition which is defined as the science of establishing the identity (set of attributes associated with a person) of an individual based on physical or behavioral characteristics of the person in either automatic or semi-automatic manner; each one of these characteristics are known as 
biometric, in particular the following factors must be considered in order to determine the suitability of the biometric for being considered as descriptive characteristic of the person: Universality (Every person must posses it), Uniqueness (different across individuals in the population), Permanence (time invariant) and Circumvention (difficult to be imitated by either impostor or artifacts). Person identification is useful in some fields such as security for access control, corpse identification, criminal investigation, missing children, parenthood determination, among others [1].

Some examples of biometrics commonly used are: fingerprint, palm print, iris, voice, face, ear structure, gait, among others [2-6] which fulfill the descriptive characteristics mentioned above. In general, a Biometric system (Figure 1) consists of the following modules:

-Sensor Module: In this module the capture of the biometric is carried out and the digital sensor used depends on the biometric to be analyzed. In the case of voice the required sensor is a microphone but in the case of other biometrics such as iris, face, gait, fingerprint and hand the sensor used is a device based on CCD (Charge-Coupled Device), particularly digital cameras are commonly used.

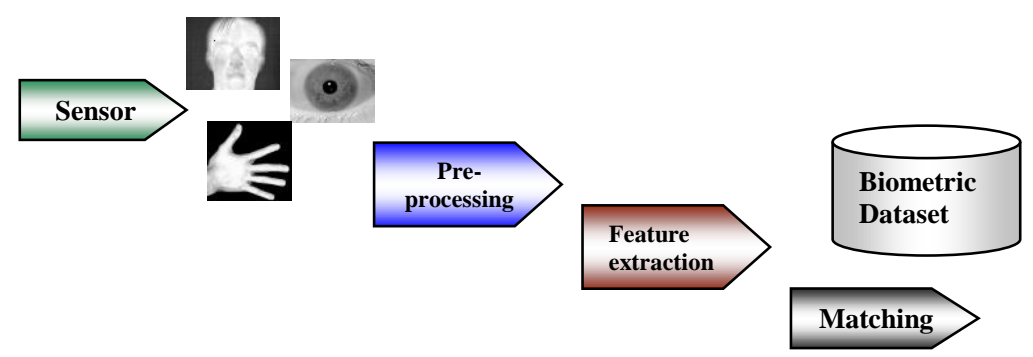

Fig. 1. Modules in a Biometric System: Sensor, Pre-processing, Feature extraction and Matching. The biometric images (face, hand, iris) were obtained from [7].

-Pre-Processing module. Once the biometric has been digitalized (captured) it is necessary to prepare the information provided by the biometric. When the biometric is captured as a digital image, typical algorithms applied in this module are focused on denoising data, enhancing contrast, equalization, rotation, among others. This preprocessing phase is fundamental in any biometric system since the better quality of the biometric information the better identification accuracy is obtained.

-Feature extraction module. This component is focused on detecting and extracting the most relevant components from the biometric which are enough descriptive for characterizing the person's identity. In particular, for the case of digital images, the first process applied in this stage is the extraction of ROI (Region of Interest) that is the sub region (from the whole image) in which the main descriptors of the biometric are located. The ROI extraction is followed by a segmentation process in order to isolate regions which indeed are relevant for biometric purposes (i.e. isolating them and discarding other non useful components in the image such as the background).

-Matching module. In this module, based on the extracted features, they are analyzed in order to determine the person's identity i.e. recognize the person. In 
biometric systems the main task in the context of recognition is to establish the association between an individual and his personal identity. For establishing this association some algorithms known as supervised classifiers are used. The classifier learns patterns from the biometric and matches them with other samples of valid users for determining whether the person's identity is accepted. In the context of supervised classifiers, this process is named classification and the database used for matching is named training set; the classifier inputs corresponds to the biometric descriptors obtained in the previous biometric modules. When the matching process is not successful then it is said that the person trying to recognize is an impostor or intruder.

In the literature, several biometrics have been analyzed for person recognition purposes but recently the hand dorsal veins also are used as biometric; in this work an approach for auto-rotating hand dorsal vein images is proposed which is applicable in the pre-processing module of any biometric system.

The content of this paper is organized as follows: Section 2 describes some relevant concepts about hand veins as biometric; in section 3 the proposed approach for rotating is described and some experimental results are presented; finally in section 4 the conclusions from this work are given.

\section{Hand Dorsal Veins as Biometric}

As mentioned in section 1, there exists several biometrics which can be considered as baseline for person recognition; recently the hand dorsal veins has been analyzed and used as biometric $[8,9]$; for capturing this biometric a digital camera is used but these kind of devices operates in the visible range $(400-700 \mathrm{~nm}$ wavelength) of the electromagnetic spectrum i.e. illuminating the region to capture using visible light. In the captured images it is difficult to discern the hand dorsal veins since in some cases the person's hand veins are not superficially visible due some corporal factors such as complexion or skin color. On the other hand, the Infrared (IR) light $(2.5-50 \mu \mathrm{m}$ wavelength) is a non visible light (for the human eye) that penetrates into the skin about $3 \mathrm{~mm}$ depth. Due to the hemoglobin properties in the veins, it absorbs the infrared radiation and as consequence, the veins are contrasted in a dark color. When IR light is used to capture dorsal vein images, it is necessary to capture via IR cameras which are known as thermal cameras and the captured images are named IR images or thermal images since the temperature (thermal) is a kind of radiation in the IR band.

The vein pattern in the dorsal hand is a vast network of blood vessels underneath a person's skin. Similarly to fingerprints, the shape of vascular patterns is different among different people and it is stable over a long period of time. In addition, as the blood vessels are hidden underneath the skin and they are mostly not visible to the human eye and as consequence they are difficult to copy by impostors. Another characteristic of the hand vein recognition is that it requires analyzing alive people because when person is not alive the vein pattern would change, also temperature, color and contrast (in the IR band).

For processing hand thermal images as initial step they must be denoised since in the IR band the images are contaminated by salt and pepper noise. In order to reduce 
the amount of noise some softening filter (mean, median, alpha-trim, Gaussian, etc.) can be applied.
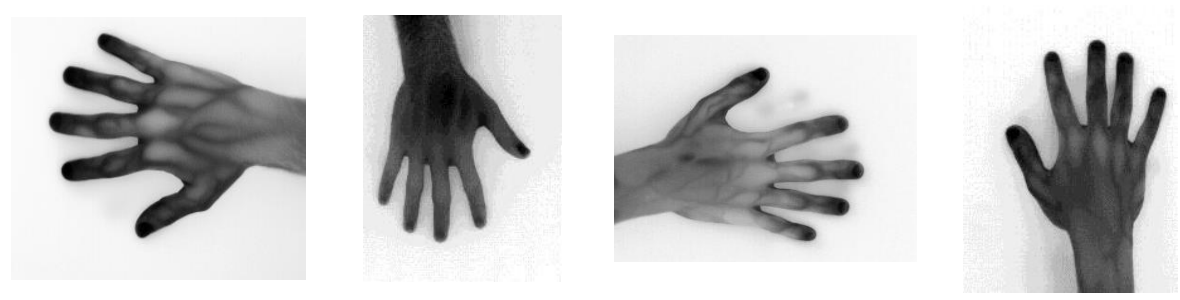

Fig. 2. Some hand thermal images where the hands were captured with different orientations [10].

Before the feature extraction step, an image rotation method must be used in order align all the images because usually when the hand images are captured, not all the hands are oriented in exactly the same direction. In figure 2, some hand images in different orientations are depicted; it can be seen than the images are not oriented in the same direction which makes difficult the ROI and feature extraction processes. Due the orientation problem, in this work we propose an approach for rotating hand thermal images in order to align them in the same direction; in the next section our approach is described.

\section{Image Rotation through Central Hand Points}

As it was stated before, the feature extraction is a crucial stage in a biometric system because the extracted features are the baseline (input) in the training set used by classifiers. In the case of hand dorsal veins as biometric the patterns learned by classifier are similar to that learned in fingerprints; the hand veins are vast net of veins interconnected therefore the bifurcations and intersections (minutiae) from them (Fig. 3 ) are distinctive, unique and different from each person to other [11-13]. Due the nature of minutiae in hand vein, the spatial description is sensible to minimal changes in rotation which impacts the feature extraction phase and an automatic image alignment method is required.

In this work, we present an auto-rotating method for thermal hand vein images; this method is named Rotation through Central Hand Points (RCHP) and its main idea consist in finding reference points from which the rotation is carried out in order to align the image. RCHP is mainly useful in the cases where there are not land marks or pivot points taken as reference to rotate an image.

RCHP finds two central points $R_{p l}=\left(x_{r 1}, y_{r 1}\right)$ and $R_{p 2}=\left(x_{r 2}, y_{r 2}\right)$ which are the central points of the hand and wrist respectively and they both are common regions in any dorsal hand image captured. Through $R_{p 1}$ and $R_{p 2}$ the reference line $\overline{R_{p 1} R_{p 2}}$ is considered to rotate the image $\theta$ degrees (Figure 4). For computing the $\theta$ value the tangent definition is used as in expression (1) where $d_{x}$ and $d_{y}$ are the differences 
between $x_{r 2}, x_{r 1}$ and $y_{r 2}, y_{r 1}$ respectively $\left(d_{x}=x_{r 2}-x_{r l}, d_{y}=y_{r 2}-y_{r l}\right)$. These differences let us know how the hand is initially oriented (Fig. 4) and it must be considered when assigning the $\theta$ value.

$$
\theta= \begin{cases}\tan ^{-1}\left(\frac{d y}{d x}\right) & \text { if }(d y \geq 0 \wedge d x \geq 0) \vee(d y<0 \wedge d x<0) \\ 180+\tan ^{-1}\left(\frac{d y}{d x}\right) & \text { if }(d y \geq 0 \wedge d x<0) \vee(d y<0 \wedge d x \geq 0)\end{cases}
$$

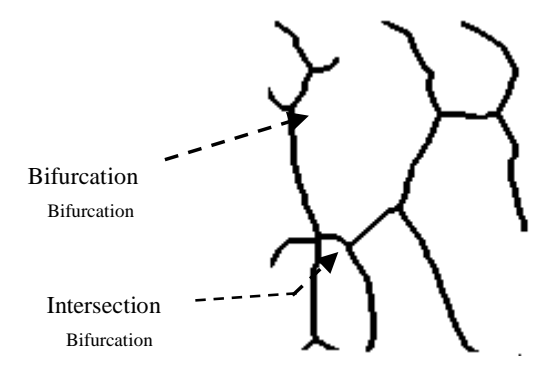

Fig. 3. Hand vein minutiae: bifurcations and intersections, which are distinctive, unique and different among people.

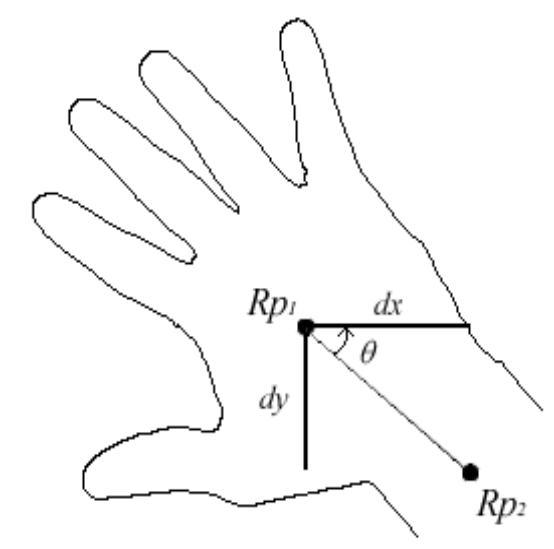

Fig. 4. Hand central reference points $R_{P 1}, R_{P 2}$ and the reference line $\overline{R_{P 1} R_{P 2}}$ used for rotating.

According to expression (1) it can be noticed that as result of the rotation, for the four cases, the image is vertical oriented i.e. the fingers will be located in the bottom region and the wrist in the top region.

In figure 5, the RCHP auto-rotation step by step process is shown. As initial step, the hand must be segmented through binarization (Fig. 5b) using as threshold the median of the gray scale in the image and a mask is obtained. The central point $R_{P I}$ of the hand (fig. $5 \mathrm{c}$ ) is computed as the mean point $\left(x_{m}, y_{m}\right)$ among all the white pixels in the mask. The central point of the wrist $R_{P 2}$ is obtained by finding the region where the wrist is located. 


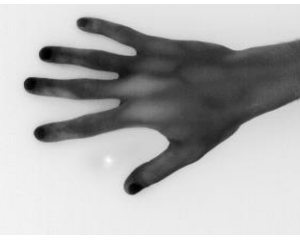

a)

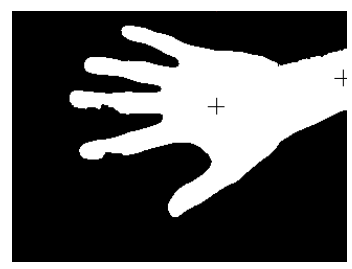

d)

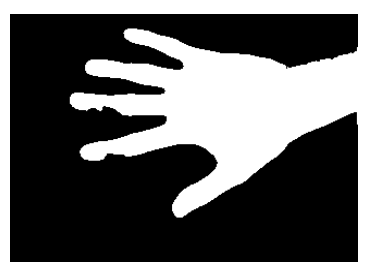

b)

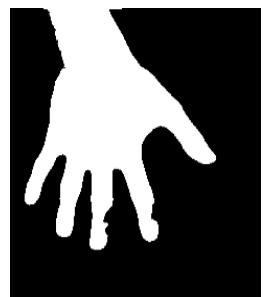

e)

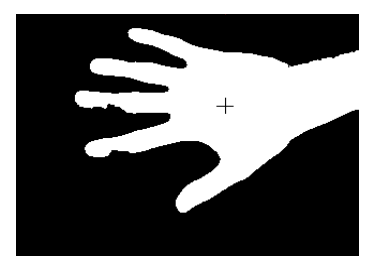

c)

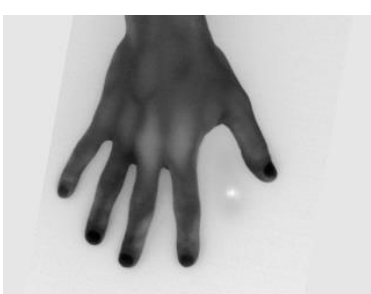

f)

Fig. 5. RCHP step by step process. a) Hand thermal image. b) Mask obtained after binarization c) Hand central point d) Hand central and wrist central points obtained. e) Mask rotated $\theta$ degrees. f) Final result obtained by RCHP.
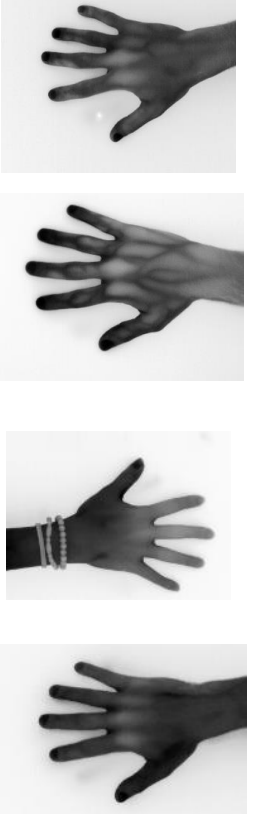

I)
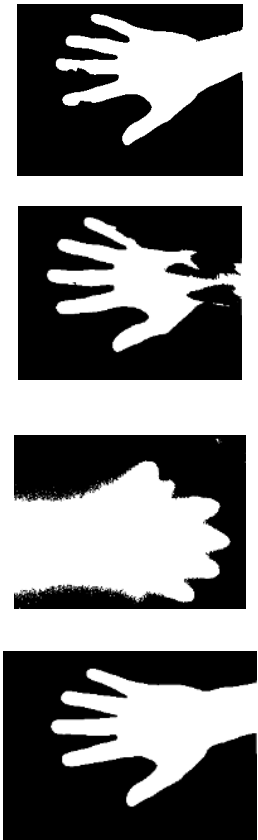

II)
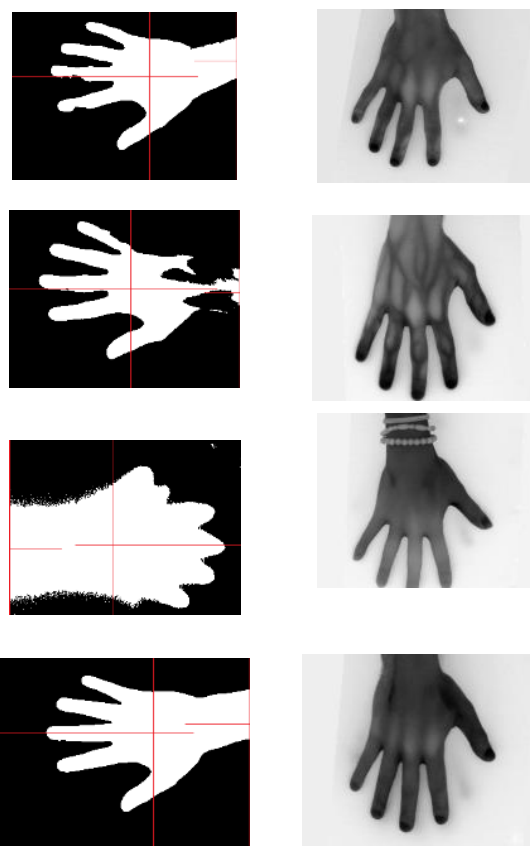

III)

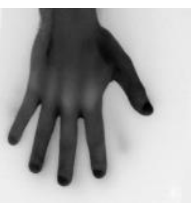

IV)

Fig. 6. Results obtained by RCHP. I) Hand thermal images. II) Central points found. III) Mask obtained alter binarization. IV) Final image rotated. 
For finding the wrist, the border of the mask is processed in order to obtain the two lateral points $l_{w 1}=\left(x_{w 1}, y_{w 1}\right), l_{w 2}=\left(x_{w 2}, y_{w 2}\right)$ that constitute the wrist; $l_{w 1}, l_{w 2}$ are found by searching the two pixel over the mask border such that they have the most white pixels connected i.e. lateral points defining the wrist and $R_{P 2}$ is approximated as the mean of the lateral points (fig 5d) using (2).

$$
R_{P 2}=\left(\frac{x_{w 1}+x_{w 2}}{2}, \frac{y_{w 1}+y_{w 2}}{2}\right)
$$

After $R_{P 2}$ and $R_{P 2}$ are obtained, the mask is rotated based on (1) and the final result of the rotation by RCHP is depicted in figures $5 \mathrm{e}$ and $5 \mathrm{f}$.

RHCP was applied over hand thermal images taken from the Technocampus dataset [10] which contains hand thermal images from 100 users and the size of each image is $320 \times 240$ pixels. The images shown in figure 2 are some examples of the images from Technocampus dataset.
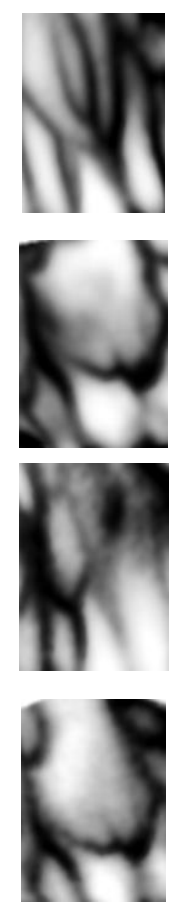

a)
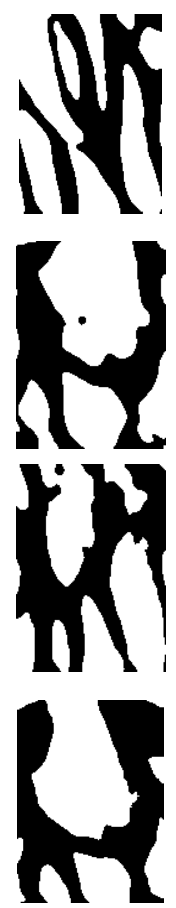

b)
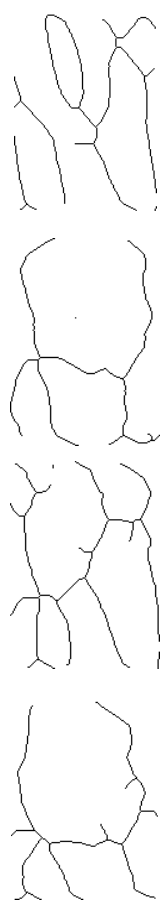

c)

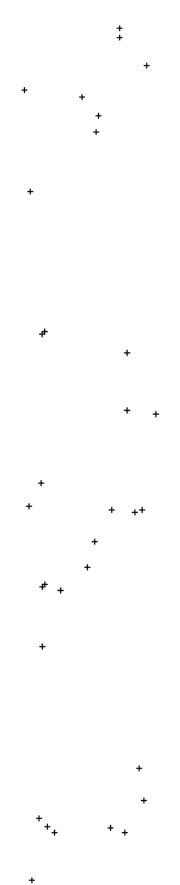

d)

Fig. 7. Minutiae extraction from hand dorsal veins. a) Hand thermal images enhanced. b) Hand veins segmented. c) Thinned veins. d) Minutiae extracted.

In figure 6 , the first column depicts the hand image to be rotated by RCHP; the second column depicts the mask found after binarization. The third and fourth columns show the central points found and the results of the rotation obtained by RCHP. Based on the experimental results, it can be seen that RCHP obtains vertical 
and homogeneous alignment from hands originally captured in different orientations. The RCHP usefulness is mainly the benefit for the feature extraction module in a biometric system because of the spatial description of the minutiae in hand vein patterns.

The next phase of the pre-processing is the minutiae extraction, as another experiment we segmented veins from images applying dynamic enhancement and 2-D Wiener filters as suggested and tested in [13, 14]. In figure $7 \mathrm{a}$, the results of vein enhancement are reported. For segmenting veins the binarization applied takes as threshold the mean and deviation of sub-regions of size $5 \times 5$ over the image; this result is depicted in figure $7 \mathrm{~b}$. Once the veins are segmented, they must be thinning to be reduced to their minimal structure representation; for thinning we used morphological operators using $3 \times 3$ identity structural element (results are shown in fig 7c). Finally, the minutiae were extracted by analyzing the neighborhood of each pixel in the thinned veins such that intersections and bifurcations have at least one neighbor defining either bifurcation or intersection forms.

\section{Conclusions}

In biometric systems one of the modules is the minutiae (feature) extraction which is a crucial phase since the minutiae are the baseline for the classifiers used in the person recognition stage. Due the kind of minutiae considered in hand dorsal veins (intersections, bifurcations) their spatial/geometric description is highly sensitive to minimal rotation, which in most of the cases is not controlled when capturing. In this work we proposed an approach named RCHP and based on our experimental results it is able to auto-rotate hand dorsal images in order to obtain images vertically oriented and as result the minutiae process is much less sensitive to the original orientation of the image. RCHP is based on finding central points of the hand and through them the rotation is carried out. A special characteristic of RCHP is that it does not require landmarks or reference points specified during the capture as parameters for autorotating hand dorsal vein images.

As future work we will evaluate the minutiae (bifurcations, intersections) extracted though RCHP rotation not only using statistical classifiers but also analyzing the hand recognition as a problem in the graph domain where the recognition is mapped to a graph matching problem therefore we will work on defining a representation of the veins features in the graph space.

Acknowledgment. This research is supported through the project OLLJ-ING15-I from VIEP-BUAP, Puebla, Mexico.

\section{References}

1. Jain, A.K., Ross, A.A., Nandakumar, K.: Introduction to Biometrics. Springer (2012)

2. Aboshosha, A., El Dahshan, K.A., Karam, E.A., Ebeid, E.A.: Score Level Fusion for Fingerprint, Iris and Face Biometrics. International Journal of Computer Applications 111(4), pp. 47-55 (2015) 
3. Xing, X., Wang, K., Lv, Z.: Fusion of Gait and Facial Features using Coupled Projections for People Identification at a Distance. Signal Processing Letters 22(12), pp. 2349-2353 (2015)

4. Menotti, D., Chiachia, G., Pinto, A., Robson Schwartz, W., Pedrini, H., Xavier Falcao, A., Rocha, A.: Deep Representations for Iris, Face, and Fingerprint Spoofing Detection. IEEE Transactions on Information Forensics and Security 10(4), pp. $864-879$ (2015)

5. Shinzaki, M., Iwashita, Y., Kurazume, R., Ogawara, K.: Gait-based person identification method using shadow biometrics for robustness to changes in the walking direction. In: IEEE Winter Conference on Applications of Computer Vision (WACV), pp. 670-677 (2015)

6. Jacob, L., Raju, G.: Ear Recognition Using Texture Features-A Novel Approach. In: Advances in Signal Processing and Intelligent Recognition Systems, pp. 1-12, Springer International Publishing (2014)

7. Computer Vision Research Laboratory dataset. University of Notre Dame. http://www3.nd.edu/ cvrl/CVRL/CVRL_Home_Page.html

8. Heenaye, M., Khan, M.: Representation of Dorsal Hand Vein Pattern Using Local Binary Patterns (LBP). In: S. El Hajji et al. (Eds.), C2SI 2015, LNCS 9084, pp. 331-341, Springer-Verlag (2015)

9. Djerouni, A., Hamada, H., Loukil, A., Berrached, N.: Dorsal Hand Vein Image Contrast Enhancement Techniques. International Journal of Computer Science Issues 11(1), pp. 137-142 (2014)

10. Faundez-Zanuy, M., Mekyska, J., Font-Aragonés, X.: A new hand image database simultaneously acquired in visible, near-infrared and thermal spectrums. Cognitive Computation 6(2), pp. 230-240 (2014)

11. Jain, A.K., Bolle, R.M., Pankatni, S.: Biometrics: Personal Identification in Networked Society. Kluwer Academic Publishers, Dordrecht (1999)

12. MacGregor, P., Welford, R.: Veincheck: imaging for security and personnel identification. Adv. Imaging 6(7), pp. 52-56 (1991)

13. Wang, L., Leedham, G., Siu-Yeung, C.D.: Minutiae feature analysis for infrared hand vein pattern biometrics. Pattern recognition, 41(3), pp. 920-929 (2008)

14. Morales-Montiel, I.I., Olvera-López, J.A., Martín Ortiz, M., Orozco-Guillén, E.E.: Hand Vein Infrared Image Segmentation for Biometric Recognition. Research in Computing Science 80, pp. 55-66 (2014) 